\title{
BMJ Open Primary care interventions and current service innovations in modifying long-term outcomes after stroke: a protocol for a scoping review
}

\author{
Dominika M Pindus, ${ }^{1}$ Lisa Lim, ${ }^{1}$ A Viona Rundell, ${ }^{1}$ Victoria Hobbs, ${ }^{1}$ \\ Noorazah Abd Aziz, ${ }^{1,2}$ Ricky Mullis, ${ }^{1}$ Jonathan Mant ${ }^{1}$
}

To cite: Pindus DM, Lim L, Rundell AV, et al. Primary care interventions and current service innovations in modifying long-term outcomes after stroke: a protocol for a scoping review. BMJ Open 2016;6: e012840. doi:10.1136/ bmjopen-2016-012840

- Prepublication history and additional material is available. To view please visit the journal (http://dx.doi.org/ 10.1136/bmjopen-2016012840).

Received 26 May 2016 Revised 25 August 2016 Accepted 20 September 2016

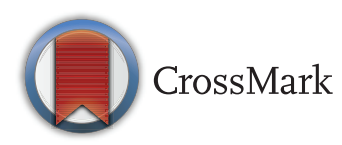

${ }^{1}$ Department of Public Health and Primary Care, University of Cambridge, Strangeways Research Laboratory, Cambridge, UK ${ }^{2}$ Department of Family Medicine, National University of Malaysia, Bandar Tun Razak Cheras, Kuala Lumpur, Malaysia

Correspondence to Dr Dominika M Pindus; D.M.Pindus@medschl.cam. ac.uk

\section{ABSTRACT}

Introduction: Interventions delivered by primary and/ or community care have the potential to reach the majority of stroke survivors and carers and offer ongoing support. However, an integrative account emerging from the reviews of interventions addressing specific long-term outcomes after stroke is lacking. The aims of the proposed scoping review are to provide an overview of: (1) primary care and community healthcare interventions by generalist healthcare professionals to stroke survivors and/or their informal carers to address long-term outcomes after stroke, (2) the scope and characteristics of interventions which were successful in addressing long-term outcomes, and (3) developments in current clinical practice.

Methods and analysis: Studies that focused on adult community dwelling stroke survivors and informal carers were included. Academic electronic databases will be searched to identify reviews of randomised controlled trials (RCTs) and controlled trials, trials from the past 5 years; reviews of observational studies. Practice exemplars from grey literature will be identified through advanced Google search. Reports, guidelines and other documents of major health organisations, clinical professional bodies, and stroke charities in the UK and internationally will be included. Two reviewers will independently screen titles, abstracts and full texts for inclusion of published literature. One reviewer will screen search results from the grey literature and identify relevant documents for inclusion. Data synthesis will include analysis of the number, type of studies, year and country of publication, a summary of intervention components/service or practice, outcomes addressed, main results (an indicator of effectiveness) and a description of included interventions.

Ethics and dissemination: The review will help identify components of care and care pathways for primary care services for stroke. By comparing the results with stroke survivors' and carers' needs identified in the literature, the review will highlight potential gaps in research and practice relevant to long-term care after stroke.

\section{Strengths and limitations of this study}

- To the best of the authors' knowledge, the proto$\mathrm{col}$ is the first to propose a comprehensive review of the literature (including unpublished examples of good practice) on primary care and community-based interventions delivered by generalist healthcare professionals to address longterm outcomes after stroke.

- Owing to the proposed scope (the inclusion of published reviews of trials, observational studies and practice examples from the grey literature, inclusive of any long-term outcome but medication efficacy; relevant to stroke survivors and carers), reviews will help identify the gaps in the evidence base and current practice. Consequently, more specific recommendations for future research and interventions can be made.

- Protocol based on a validated methodological approach and systematic search strategy.

- Supported and guided by stakeholder involvement (healthcare professionals, patient representative, planned consultations with patient advisory panel including caregivers) from the conception of the protocol to knowledge dissemination.

- Owing to the breadth of coverage, the review will focus on reviews of the literature rather than original studies (although most recent trials will be reviewed).

\section{INTRODUCTION}

As survival after stroke improves, ${ }^{12}$ the longer term care of people with stroke is going to play an increasingly important part of stroke care. Surveys demonstrate that the longer term needs of people with stroke and their informal carers are not being adequately addressed and that the majority of stroke survivors are dissatisfied with care after discharge from hospital. ${ }^{3}{ }^{4}$ Two major themes that run through these surveys are information needs 
(54\% reported wanting more information about stroke) and feelings of abandonment (42\% reported feeling abandoned after leaving hospital $\left.{ }^{3}\right)$. Other important themes include the emotional and social impact of stroke in addition to the many physical and cognitive consequences including problems with mobility, memory, concentration and fatigue. ${ }^{5}$

The evidence of effectiveness of interventions and services aimed at the long-term care of stroke survivors is sparse ${ }^{6}$ especially beyond the first year after stroke. ${ }^{7}$ In the UK, initial 6-week, 6-month and thereafter annual follow-up assessments constitute the only recommended formal mechanism of comprehensive long-term care. ${ }^{4}$ Nonetheless, recent reports estimated that $39 \%$ of stroke survivors do not receive a follow-up assessment ${ }^{8}$ and only $17 \%$ have one at 6 months. ${ }^{9}$ Thus, the long-term care of stroke survivors is largely taken on by ad hoc opportunistic, primary and community healthcare services after hospital discharge.

Stroke results in a wide range of disability, ${ }^{10}$ which requires complex interventions ${ }^{11}$ to address functional and psychosocial outcomes. ${ }^{12}{ }^{13}$ These functional and psychosocial consequences of stroke persist beyond the initial period of hospital rehabilitation, ${ }^{11}{ }^{14}$ creating long-term needs, ${ }^{3}$ which require further support in the community. Several reviews of interventions addressing specific long-term outcomes after stroke have been published, including secondary prevention, ${ }^{15}{ }^{16}$ information needs, ${ }^{17}$ physical, ${ }^{18}{ }^{19}$ psychological ${ }^{20-25}$ and cognitive rehabilitation. ${ }^{26} 27$ However, to the best of the authors' knowledge, an integrative account of these reviews summarising overall care is lacking. ${ }^{15} 17$

The involvement of acute specialist services in longterm stroke care is often limited to the first days up to a few weeks after hospital discharge. ${ }^{8}$ In contrast, primary care and community healthcare services assume longterm holistic care of patients. ${ }^{28}$ Primary care can be defined as 'the first level of contact with formal health services', which provide first contact and ongoing care for patients with all types of health problems, including stroke. $^{29}$ It may be delivered in the community or primary care settings, and primary care services can include family practice, general practice and ambulatory care settings. ${ }^{30}$ Community health services can be defined as services usually supplied by district nurses and allied healthcare professionals in the community such as physiotherapy, occupational therapy, speech and language therapy. ${ }^{31}$ Community health services can also include social services ${ }^{32}$ and psychological therapies (eg, counselling). Interventions delivered within these generalist settings have the greatest potential of reaching the majority of stroke survivors. In contrast, specialist services such as early supported discharge and neurorehabilitation services are time limited, not available in all areas (eg, in hospitals without specialist stroke units and/or rural areas) and often include specific inclusion criteria. ${ }^{33}$ For example, early supported discharge services are limited to the first few weeks poststroke and delivered to selected (estimated median of 34\%) groups of patients with stroke based on factors such as persisting need, function, stability of the condition and/or area of reach. ${ }^{33}$ Consequently, interventions delivered within primary care rather than specialist services have the greatest potential of reaching the majority of community dwelling stroke survivors. Furthermore, given the epidemiology of multimorbidity in the community, it is unlikely that a model that requires specialist long-term support for all stroke survivors would be sustainable. ${ }^{34}$

One-third of stroke survivors are functionally dependent on others and of those, one in five are cared for by their family and friends. ${ }^{35} 36$ These informal carers act as mediators in the care pathway and have their own unique needs. ${ }^{37-41}$ However, ${ }^{42}$ three quarters of informal carers feel ill-prepared for their caregiving role, ${ }^{42}$ with high numbers reporting stress $(69 \%)$, anxiety $(79 \%)$ and frustration $(84 \%) .{ }^{42}$ Poor coping and negative emotional responses to caregiving can lead to the severance of family relationships and abandonment of a caregiving role, ${ }^{43}{ }^{44}$ leading to an increased burden placed on community health services. Therefore, effective interventions to support informal carers may have an important role in meeting daily care needs of stroke survivors and helping them to remain in the community. With better understanding of how to address the longer term needs, primary care could improve health and care for both stroke survivors and their informal carers.

\section{Aims and objectives}

We therefore aim to address this gap in the literature and (1) to provide an overview of interventions delivered in primary care and community healthcare services to stroke survivors and/or their informal carers in order to address long-term outcomes after stroke; (2) to provide an integrative account of the scope and the characteristics of interventions which were successful in addressing long-term physical and/or psychosocial outcomes after stroke in either stroke survivors and/or their informal carers; and (3) to provide an overview and examples of developments in current clinical practice which are not subject to formal evaluation.

To cover the breadth of the literature, we will focus on the systematic reviews and meta-analyses of observational studies and randomised controlled trials (RCTs) and controlled trials ${ }^{45}$ of interventions delivered in primary care and community care settings to stroke survivors and their informal carers. We will complement the review of reviews with that of the most recent trials published in the past 5 years. Since we aim to identify interventions which were found effective (ie, which reported a significant change in their primary and/or secondary outcomes which are relevant to our review questions), we chose to focus on systematic reviews and meta-analyses which provide a quantitative review of findings. We recognise that many service innovations are not subjected to rigorous evaluation, but nevertheless may provide useful examples of the way in which primary 
care services might be developed. Therefore, we will also review current developments in clinical practice in relation to long-term primary and community care after stroke identified in the grey literature. We adapted the Luxemburg definition of grey literature as 'that which is produced on all levels of government, charity and international organisations in electronic formats, but which is not controlled by commercial publishers'. ${ }^{46}$ The definition encompasses the documents produced by these organisations as well as websites and online materials.

Our specific objectives are:

1. To provide an overview of the scope and focus of healthcare interventions delivered in primary care and in the community by generalist healthcare professionals to address long-term outcomes (eg, functional and psychosocial) after stroke aimed at stroke survivors and their informal carers;

2. To understand the content (eg, approach, techniques) of interventions which have been found effective by reviewed research;

3. To identify the gaps in research in addressing longterm outcomes after stroke;

4. To provide an overview of current developments in clinical practice to address the long-term needs after stroke which have been delivered in primary care and the community.

This scoping review will inform the development of primary care services to address the long-term outcomes and needs of stroke survivors and their informal carers living in the community. ${ }^{46}$

\section{METHODS AND ANALYSIS}

We have chosen a scoping review methodology, rather than a systematic review of reviews, as it offers a broader perspective of the issues. ${ }^{47}{ }^{48}$ Rather than focusing on formal evaluation of the effectiveness, the scoping methodology will help us explore what interventions and services are currently available to stroke survivors and informal carers across diverse long-term outcomes, and what we can learn from the characteristics of interventions which reported positive outcomes. We chose not to limit the scope of the review to services directed at particular outcomes as in part we aim to explore what outcomes services are directed at and whether there are any gaps (review questions 1 and 3).

The review will focus on care delivered in primary care and community healthcare settings by generalist healthcare professionals. ${ }^{29-32} 49$ We will focus on care delivered by generalist healthcare professionals as they are responsible for most long-term care of stroke survivors and informal carers. We have adopted the definition of the International Standard Classification of Occupations 2008 (ISCO-2008; ${ }^{50}$ ) of generalist and specialist medical professionals to encompass allied healthcare professions. Consequently, we defined generalist healthcare professionals as those who “(...) do not limit their practice to certain disease categories or methods of treatment (...)", and who can provide continuing care to individuals and their families. ${ }^{50}$ These healthcare professionals can include but are not limited to: general practitioners, primary care nurses, community occupational therapists and physiotherapists and social workers. We defined specialist healthcare professionals as those who specialise in certain disease categories, types of patients or methods of treatment and who diagnose, treat and prevent illness (disease, disease, injury, and other physical and mental impairments in humans), using specialised tests and diagnostic procedures. ${ }^{50}$ For example, a physiotherapist working as part of a neurorehabilitation team delivering care to patients with neurological conditions would be considered a specialist under this definition. In contrast, a physiotherapist working in the community seeing patients with differing needs, not necessarily related to any single disease category, would be considered a generalist healthcare professional.

We purposefully chose not to limit our search strategy to any specific long-term outcomes after stroke as our goal is to capture the literature relevant to physical, psychological and social needs after stroke. The long-term outcomes after stroke will be framed within the WHO International Classification of Functioning, Disability and Health (ICF) core sets for stroke ${ }^{51}$ as described in detail in Stage I: Identifying the research question.

The scoping review protocol follows the six steps approach as outlined by Arksey and $\mathrm{O}^{\prime} \mathrm{Malley}^{47}$ and further refined by Levac et al. ${ }^{52}$

\section{Stage 1: Identifying the research question}

Our scoping review aims to answer the following questions: (1) which long-term outcomes after stroke have been addressed by (a) trial, (b) observational and (c) grey literature? (2) What are the characteristics of interventions which have been identified as effective in systematic reviews and meta-analyses? (3) Which outcomes have not been currently addressed by the trial literature compared with the needs identified by stroke survivors in national surveys? And (4) what are the characteristics of examples of good clinical practice in relation to improving long-term outcomes after stroke? We did not constrain the long-term outcomes to a specific time frame after stroke (eg, 6 months poststroke). Using the WHO ICF core sets for stroke $\mathrm{s}^{51}$ as a reference framework, we will focus on outcomes which relate to (1) body functions (which include psychological functions), (2) activities and (3) participation. Within ICF core sets for stroke, body functions are broadly defined as mental and movement-related, neuromuscular, sensory and pain functions, voice and speech, and functions related to bodily systems (cardiovascular, metabolic, digestive, respiratory, etc). ${ }^{51}$ Activities and participation include mobility, communication, learning and applying knowledge, self-care, interpersonal interactions and relationships, domestic, community, social and civic life. ${ }^{51}$ We will further provide an overview of current practice and 
its associations with long-term outcomes after stroke. We will also present recent developments in good clinical practice in long-term care after stroke by reviewing practice exemplars from the UK and internationally.

\section{Stage 2: Identifying relevant literature}

\section{Review of reviews of RCTs and observational studies review} of most recent controlled trials

As presented in table 1 which reviews specific inclusion and exclusion criteria, our search strategy has been developed to identify (1) reviews of RCTs and controlled trials of primary and/or community care interventions to improve long-term outcomes after stroke in stroke survivors and/or informal carers, (2) recent RCTs and controlled trials published in the past 5 years (since January 2011); and (3) reviews of observational studies. We will focus on systematic reviews and meta-analyses as they provide a quantitative review of findings. The reviews of observational studies will be identified together with the reviews of RCTs and controlled trials using an additional inclusion criterion specific to observational designs (eg, including cross-sectional studies, longitudinal studies, retrospective cohort). We will search the five databases as recommended by the Centre for Reviews and Dissemination $^{53}$ as the basis for systematic reviews in healthcare research: MEDLINE, EMBASE, PsycINFO,
CINAHL and either Cochrane Reviews (to search for review literature) or Clinicaltrials.gov (for recent trials).

\section{Grey literature}

Little guidance exists on methods to review grey literature. Some publications focus on systematic review methods adapted for grey literature search. ${ }^{54-57}$ However, their purpose was to complement systematic reviews of published research in their respective areas. In contrast, our aim is to complement the scoping review of the trial and observational literature by identifying potential examples of good practice to inform new models of primary care. Consequently, our search strategy focuses on examples of good practice by searching the websites of professional bodies relevant to healthcare and/or stroke. Grey literature will be identified using an advanced option in google.com search engine, with limits set to site (eg, http://www.nao.org.uk), language (English) and the past 15 years to keep the search manageable and relevant to current healthcare. This strategy offers consistency compared with the reliance on varying search algorithms employed by specific websites. We will search the content of major health organisations, clinical professional bodies, and stroke charities in the UK and internationally, including but not exclusively the: (1) Stroke Association, (2) Department of Health in

Table 1 Inclusion and exclusion criteria

\begin{tabular}{|c|c|c|}
\hline Language & $\begin{array}{l}\text { Inclusion } \\
\text { English }\end{array}$ & $\begin{array}{l}\text { Exclusion } \\
\text { Any other language }\end{array}$ \\
\hline $\begin{array}{l}\text { Types of } \\
\text { studies }\end{array}$ & $\begin{array}{l}\text { Individual trials: The trials published in the past } \\
5 \text { years (since } 1 \text { January 2011) will be included. }\end{array}$ & $\begin{array}{l}\text { Qualitative reviews or a mixed-methods review, } \\
\text { where data from the RCTs were not separately } \\
\text { reported. } \\
\text { Observational studies, non-controlled studies, before } \\
\text { and after studies. } \\
\text { Conference abstracts, editorials and other } \\
\text { publications, which do not include primary research. }\end{array}$ \\
\hline $\begin{array}{l}\text { Patient } \\
\text { population }\end{array}$ & $\begin{array}{l}\text { Adult community dwelling stroke survivors and } \\
\text { informal carers }\end{array}$ & $\begin{array}{l}\text { Patient populations other than stroke. } \\
\text { Mixed-patient populations where data were not } \\
\text { analysed separately for stroke survivors. }\end{array}$ \\
\hline Interventions & $\begin{array}{l}\text { 1. Conducted within the primary and/or community } \\
\text { care ambulatory settings designed to change } \\
\text { long-term outcomes after stroke. } \\
\text { 2. Delivered by a generalist healthcare professional. }\end{array}$ & $\begin{array}{l}\text { 1. Conducted outside of the primary or community } \\
\text { care setting, including secondary and tertiary care } \\
\text { settings. } \\
\text { 2. The intervention was conducted in secondary and } \\
\text { primary care and the effects of the intervention } \\
\text { within primary care cannot be separated. } \\
\text { 3. Studies conducted in nursing homes or residential } \\
\text { care settings or in inpatient settings (eg, } \\
\text { community hospitals). }\end{array}$ \\
\hline Control group & $\begin{array}{l}\text { Any control group including usual care, no } \\
\text { intervention or attention control. }\end{array}$ & Studies without a control group will be excluded. \\
\hline Outcome & $\begin{array}{l}\text { 1. Any long-term outcome after stroke which can be } \\
\text { modified by interventions delivered within a } \\
\text { primary and/or community care setting. } \\
\text { 2. Any long-term outcome in carers of stroke } \\
\text { survivors which can be modified within a primary } \\
\text { and/or community care setting. }\end{array}$ & $\begin{array}{l}\text { Interventions focused on drug efficacy will be } \\
\text { excluded. Those focused on adherence will be } \\
\text { included. }\end{array}$ \\
\hline
\end{tabular}


the UK, (3) WHO, (4) National Institute for Health and Care Excellence, (5) UK National Audit Office, (6) National Stroke Association (USA), (7) World Stroke Organisation, (8) European Stroke Network and (9) Internet Stroke Centre (USA).

One reviewer will screen the titles and the brief text underneath for relevant documents. Links identified as relevant will be screened at the same time as titles for relevant content (figure 1). Potentially relevant documents will be saved for further screening. To keep the search manageable, we will limit the screening to the first 10 pages of results ( 100 results) relying on Google's PageRank algorithm which prioritises relevant sites. ${ }^{57}$ We chose to limit our search to the past 15 years to both manage the number of retrieved records and to focus on the results which are most relevant to the current organisation of healthcare system(s) and the interaction between different components of care. ${ }^{58}$

\section{Database search strategy}

Identification of trial and observational literature: reviews

We have developed a set of key terms organised according to the study type and Population Intervention Comparison and Outcome (PICO) framework: (1) Population: terms related to stroke (eg, stroke*, 'cerebrovascular accident*', CVA) were triangulated with

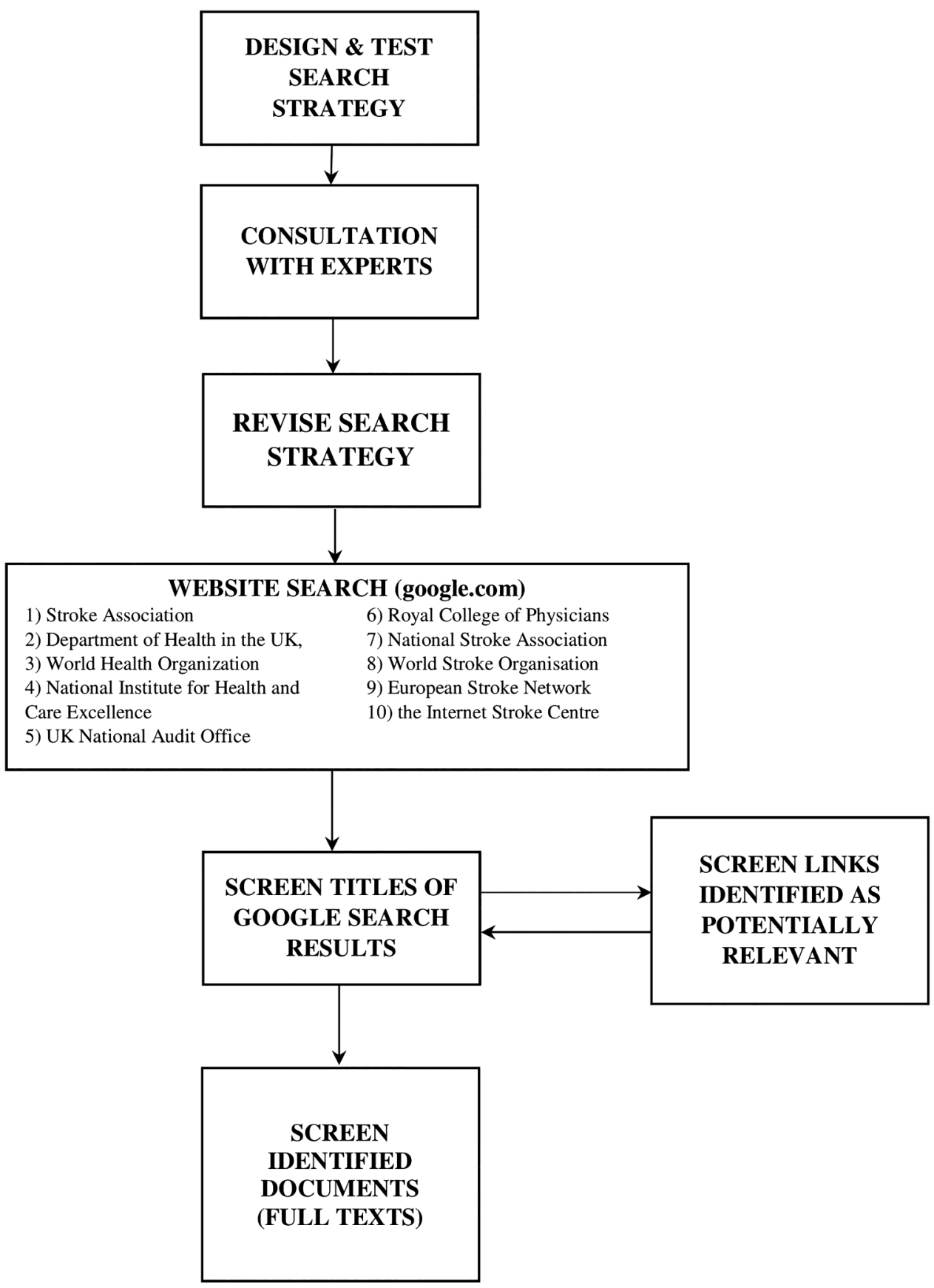

Figure 1 A flow diagram of search strategy for grey literature. 
Boolean Operator 'AND' with those related to a stroke survivor (eg, survivor, patient*) or caregivers (eg, carer*, caregiver*, family), (2) Intervention: primary care and community health services (eg, primary care, general practice, community care/healthcare, postdischarge services, long-term care/rehabilitation), (3) Comparison: we will include any control group, (4) Outcome: any modifiable long-term outcome after stroke except for medication efficacy. The terms across the PICO categories will be triangulated with Boolean Operator AND. We will focus on review literature searched using a filter adapted from the Scottish Intercollegiate Guidelines Network for systematic reviews ${ }^{59}$ and adapted to include non-systematic reviews. Text within the title or abstract, and Medical Subject Headings (MeSH) term (or equivalent) searches will be conducted. An example of a search strategy developed for MEDLINE is included in online supplementary appendix 1 . We will only include studies which focus on the population of community dwelling stroke survivors and on care delivered within ambulatory primary care and community healthcare settings (table 1). Studies conducted in nursing homes, residential care settings or in inpatient settings (eg, community hospitals) will be excluded. No time or language limits will be set but due to pragmatic reasons only texts in English will be included. We will only include published and peer-reviewed reviews. Reviews of observational studies will be identified together with the reviews of trial literature and sorted based on the design of included studies.

The development of the search strategy was iterative and followed three steps. In the first step, a preliminary set of key terms was developed based on previous literature and key words identified in a qualitative review protocol conducted within our team. ${ }^{49}$ In the second step, we conducted a preliminary search in PubMed. The search strategy and key terms identified were further reviewed by our research team. Key terms were expanded (eg, we added 'general practice' and 'general practitioner'), terms related to specific outcomes were excluded, and those related to the population of interest (eg, stroke survivors, patient, caregiver) were included. In the third step, the final search strategy was employed and adjusted for each of the specific databases. Search strategies for each of the five databases were reviewed by a medical librarian experienced in systematic review methods. Further eligibility criteria for the studies to be included in the scoping review are described in table 1.

\section{Identification of trial literature: most recent trials}

Using the same set of key terms triangulated with a filter to identify controlled ${ }^{60}$ and RCTs ${ }^{61}$ (see online supplementary appendix 2), we will search five databases (MEDLINE, EMBASE, PsycINFO, CINAHL, Clinicaltrials. gov) for trials published in the past 5 years (since 1 January 2011). The combination of filters developed by the Cochrane Collaboration for RCTs and Robinson et $a l^{60}$ was used to maximise sensitivity while also preserving specificity ${ }^{62}$ The search strategy has been tested in MEDLINE and reviewed by a medical librarian. Following the screening of abstracts, we will crossreference the identified titles against the reference lists of the included reviews. Such a strategy will help identify trials assessing a wide range of long-term outcomes after stroke, thus supplementing the review of reviews. Given that we are interested in a broad range of long-term outcomes after stroke, we made a pragmatic choice to narrow the search of the most recent trials to the past 5 years. As with review literature, we will only include published and peer reviewed articles written in English.

\section{Identification of grey literature}

A search strategy for the review of grey literature was developed iteratively. First, initial key terms were identified based on the peer reviewed trial literature, which was then tested against specific websites (Department of Health and Stroke Association) as well as Google search using advanced settings. The hand search of the Stroke Association and Department of Health websites helped narrow the terms related to stroke and primary care. The strategy was then reviewed by an interdisciplinary group of experts (allied healthcare professionals, academics specialising in general practice and stroke and a stroke survivor) who suggested adding key terms of 'best practice' and 'effective service delivery', and the inclusion of additional websites: for example, National Audit Office and Royal College of Physicians.

We developed three broad groups of search terms: (1) examples of good practice (eg, 'good practice', 'best practice', 'effective service delivery', 'practice example'), (2) the context of primary care and community services (eg, 'general practice', 'community health services', 'voluntary service', 'charity worker', 'social services') and (3) stroke. A set of key terms for each of these categories will be triangulated using Boolean Logical Operators AND/OR. We reduced stroke-related search terms to a single term 'stroke', as this term was used most frequently in relation to healthcare services in general Google search. An example of the grey literature search strategy developed for the National Audit Office is included in online supplementary appendix 3 . We set language limits to English only and, depending on the breadth of the website, set a pragmatic time limit to 1 January 2000.

\section{Inclusion and exclusion criteria}

Specific inclusion and exclusion criteria are listed in table 1 . We will only include evidence on interventions to modify outcomes which are addressed within a primary and/or community care setting by a generalist healthcare professional. For example, we will include outcomes to improve mobility after stroke which would not require specialist neurorehabilitation knowledge, and could therefore be delivered by a community-based physiotherapist. In contrast, cognitive rehabilitation delivered by a neuropsychologist as part of a 
neurorehabilitation programme would be considered outside the scope of this review. In relation to secondary prevention, we will include evidence on medication adherence but trials and reviews of medication efficacy will be excluded. We will address the long-term needs of carers including emotional, psychological and health outcomes.

\section{Stage 3: Study selection}

Review of reviews of RCTs and observational studies, review of most recent controlled trials

Data will first be downloaded to EndNote X7.1 (EndNote X7.1, Thomson Reuters). Titles will be exported to an Excel spreadsheet where the screening results will be recorded. In phase 1 (review of reviews of trials), two reviewers will independently screen the titles of reviews identified by our search strategy (figure 2). Next, we will screen abstracts of potentially relevant reviews using our inclusion/exclusion criteria (table 1). In the final stage of phase 1 , two reviewers will screen the full texts using the data extraction spreadsheet (available on request) to extract the information listed in table 2 onto an Excel spreadsheet. Any inconsistencies across all stages will be resolved by discussion between the reviewers. An experienced third reviewer will be consulted should no consensus be reached. The second phase (RCTs and controlled trials published in the past 5 years) will follow the same process as described above. The review of reviews of observational studies will follow the strategy as outlined for the trial literature except for the inclusion criteria related to study type and control groups. Data will be managed in the same manner as the data for the trial literature. We will include observational studies (eg, cross-sectional, longitudinal, retrospective cohort), which focus on primary and community care after stroke. We will use the PRISMA flow diagram to report the number of screened and included studies.

\section{Grey literature review}

Grey literature search will be performed by one reviewer. A reviewer will screen the results of the Google search (titles and brief text underneath the title) to identify potential documents with examples of good practice within primary and community care relevant to longterm outcomes after stroke. Documents deemed relevant will be downloaded for data extraction and record keeping. We will operationally define the examples of good practice as case studies in guides, guidelines, action plans, consultation papers developed by government agencies, local and national health authorities and professional bodies (eg, National Audit Office Good Practice Guide, policy documents on transforming community services from the UK Department of Health, Royal College of Physicians Sentinel Stroke National Audit Programme (SSNAP) Report). The documents identified as relevant will be saved for further screening using the following inclusion criteria: (1) the practice

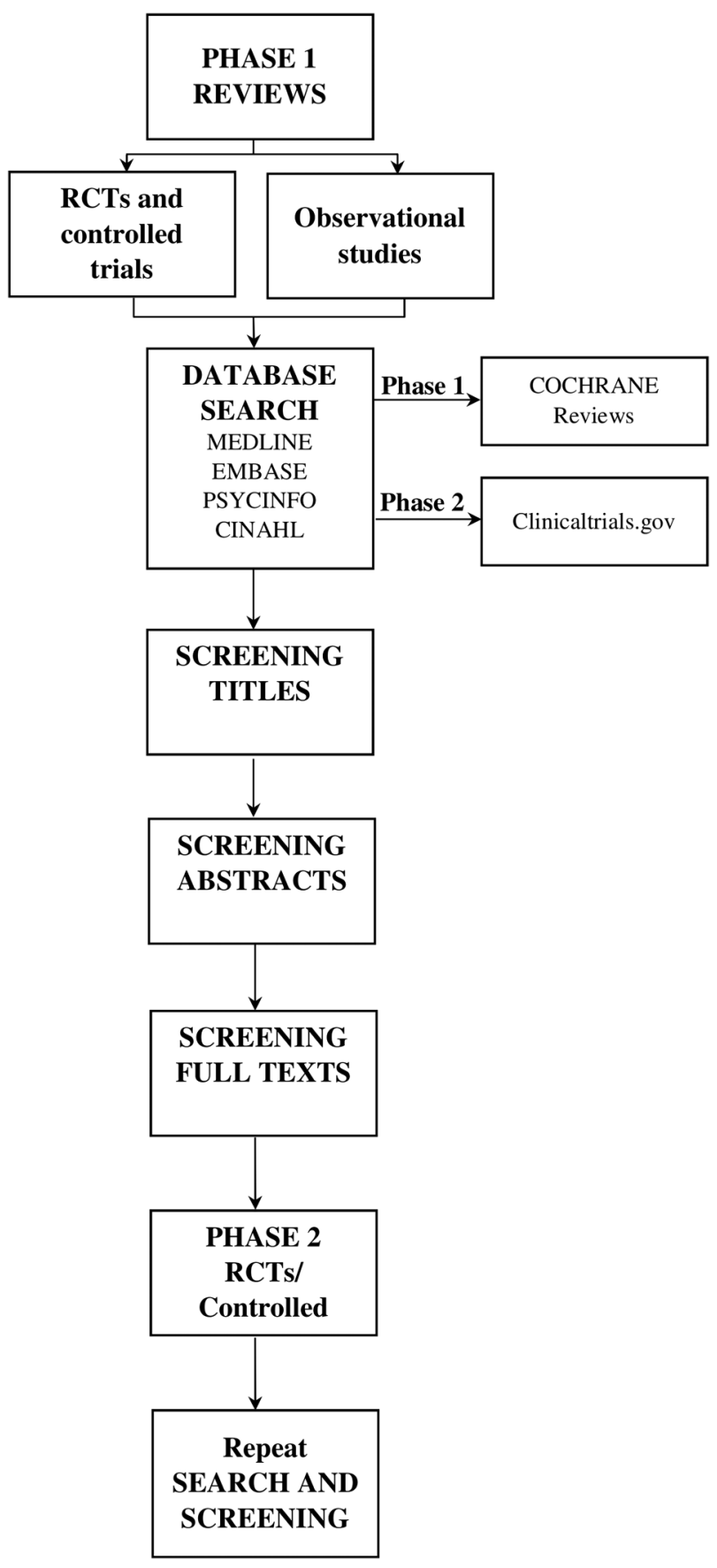

Figure 2 A flow diagram of search strategy for trial literature and observational studies. RCTs, randomised controlled trials.

example has to focus on care after stroke (for a survivor and/or informal carer), (2) on improving long-term outcomes, (3) it has to be delivered by general practice, (4) practice components have to be clearly described including information on what was delivered, by whom and to whom, and its outcomes, and (5) the justification for why it was considered good practice has to be provided. The identified examples of good practice will be reviewed and discussed within the research team and as part of the consultation exercise to assess their utility for a new model of primary care. 
Table 2 An example of the data extraction framework for the review of trial literature

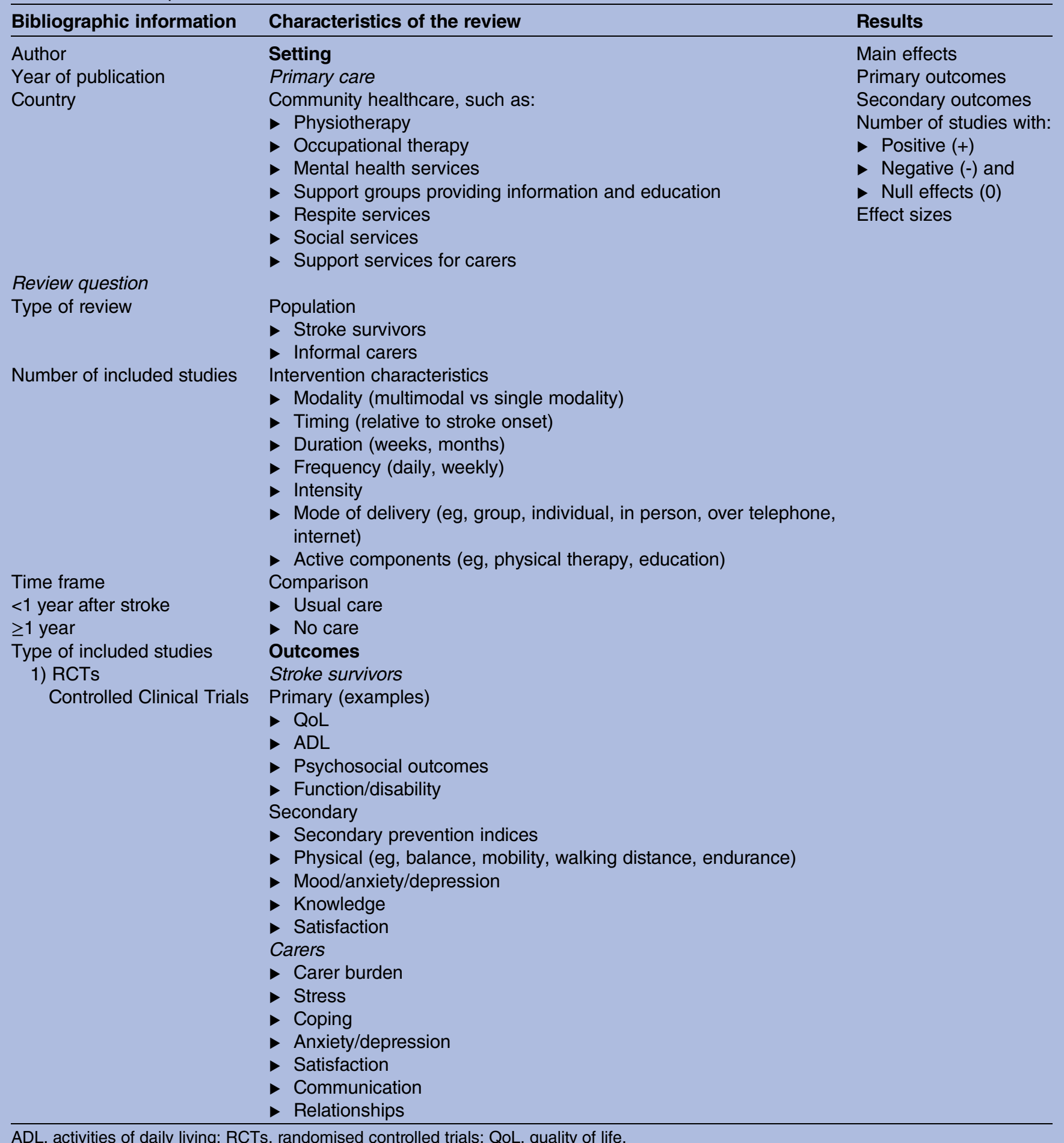

$\mathrm{ADL}$, activities of daily living; RCTs, randomised controlled trials; QoL, quality of life.

Stage 4: Charting the data

RCTs and controlled trials

We will summarise the information from reviews and most recent experimental research using descriptive methods. On the basis of the scoping review objectives, we have developed a data extraction framework to help visualise the key outcomes to answer our research questions (table 2). The review data chart will include: (1) bibliographic information, (2) characteristics of the review (eg, setting, population, intervention characteristics, outcomes), and (3) main results (summary of the reported effects and effects sizes). The data chart will summarise the key characteristics of interventions (timing, duration, frequency, 'active ingredients', mode of delivery and who delivered the intervention) in relation to specific outcomes. It will also help identify potential gaps in research in relation to the type and timing of interventions (eg, within the first year since stroke or 
beyond), its scope and the long-term outcomes it addressed (eg, psychological outcomes, those related to physical function, participation, activities). Since the methodology of a scoping review is guided by iterative approach to data synthesis, ${ }^{48}$ we will first develop a preliminary data extraction sheet based on the chart presented in table 2. The data extraction form will then be pilot tested by one reviewer, consulted with the second reviewer and, on reaching the consensus, finalised. Since no formal quantitative synthesis will be attempted, we will rely solely on the data reported by the authors in publications. Tabulation of results from the RCTs and controlled trials using similar categories and following the same process as that described for the reviews will aid comparative analyses of the evidence from phases 1 (reviews) and 2 (most recent RCTs and controlled trials).

\section{Observational studies and grey literature}

First, the results from the reviews of observational studies will be charted using an adapted data extraction framework developed for the reviews of RCTs (table 2). As in the review of trial literature, the development of a data extraction sheet will be iterative. The characteristics of primary and/or community care services (eg, service provider, target population, service availability and/or use, timing of service and, where appropriate, its duration, frequency and intensity) will be extracted. Information on the strength and the direction of the associations between service characteristics and long-term outcomes after stroke as well as study design (eg, prospective, retrospective, cross-sectional) will also be recorded.

Second, we will extract the data from grey literature focusing on the examples of good practice (eg, new service or assessment addressing a particular long-term need after stroke, refinement of existing services), the description of practice or service (eg, what was delivered and how?), service provider, timing of delivery (in relation to stroke onset), its impact on long-term outcomes and justification as to why it was considered 'good practice' (eg, benefits to stroke survivors/informal carers, larger community, adherence and/or implementation of guidelines). The data will be extracted using a data extraction form (an example is presented in online supplementary appendix 4; the form will be tested and amended once relevant literature is identified) and recorded in an Excel spreadsheet.

\section{Stage 5: Collating, summarising and reporting the results} Review of reviews of trial and observational literature, most recent trials and grey literature

In the first instance, the results of phase 1 of the review will be summarised. We aim to report which long-term outcomes have been addressed by the interventions included in our review, which areas of research (and care) are well developed and could therefore inform models of primary care services to support the long-term care of stroke survivors and carers, and which areas need further research. The discussion of findings from phase
2 will complement findings from phase 1 by mapping the areas of current focus within research and practice. We will reflect on whether and how the trials address the gaps identified in phase 1 . On the basis of findings from phases 1 and 2, we will discuss the characteristics of interventions found effective and contrast them with those which were found less effective. We will also outline the areas where further research is most needed to address needs reported by stroke survivors and caregivers in the literature. Further, using the findings from grey literature, we will provide an overview of potential components of good clinical practice to further inform pathways within a new model of primary care services. The components of clinical practice with supporting evidence will be charted against the gaps identified by the trial literature. For example, if one of the gaps identified is training for carers, we will provide details of how this might have been addressed in observational research and/or practice exemplar(s) from grey literature. On the basis of this comparison, we will be able to make specific recommendations for future research.

\section{Stage 6: Consultation exercise}

We will seek feedback on the preliminary results of the scoping reviews from stakeholders, including healthcare professionals, stroke survivors and informal carers. This will inform our interpretation of the literature, and may potentially identify additional pieces of evidence (such as ongoing trials, case studies and policy documents) to contribute to the grey literature component.

We will consult a group of healthcare professionals involved in the care of stroke survivors, including stroke specialists, general practitioners, practice nurses, allied healthcare professionals (community physiotherapists, occupational therapists, speech and language therapists and psychologists). This will take the form of a presentation followed by a discussion of the results from the scoping reviews with an advisory interdisciplinary panel of healthcare professionals involved as the Intervention Development Group in our wider programme of research. The consultation exercise will be conducted to address the following questions: (1) what long-term needs of stroke survivors and informal carers have not been addressed by the current literature? (2) Are there ongoing RCTs or controlled trials which may help address some of these needs (if yes, which needs are they addressing)? (3) Does current research (in its scope and emphasis) reflect the priorities in the long-term management of stroke? (4) What priorities for future research would inform primary and community care practice and services to manage long-term outcomes after stroke? And (5) which of the identified examples of good practice could be scaled up and adopted nationally?

The second consultation exercise will be conducted with stroke survivors and carers engaged in Patient Participation Groups. Patient Participation Groups have been chosen as they have experience in assisting researchers in reviewing their findings and can act as 
advocates for other stroke survivors. The lay summary of findings will be prepared with open questions on the relevance of the findings to current and ideal patient care, reflection on the gaps in evidence in relation to unaddressed needs after stroke, and recommendations for future research.

\section{ETHICS AND DISSEMINATION}

Our aim is to provide an integrative account of currently available interventions to address long-term outcomes after stroke in relation to survivors' and carers' longterm needs, and to characterise the interventions which were successful in addressing these outcomes. We expect several outputs from the scoping review: (1) a journal publication in the field of stroke rehabilitation and care, (2) presentation at an international conference on stroke treatment and care, (3) a consultation with a patient advisory group and (4) a consultation with an interdisciplinary group of healthcare professionals on how the findings from the scoping reviews could inform the development of a new model of primary care for stroke survivors and carers.

In conclusion, the scoping review is a critical step in identifying the gaps in current research and practice in relation to long-term outcomes after stroke. The outputs from this review will help address the gaps in current literature by providing an integrative account of available interventions. The knowledge gained from the scoping review will inform a new model of primary care for stroke survivors and carers, and will be disseminated to a wide group of stakeholders to inform practice.

Contributors DMP drafted the protocol and designed the search strategy and data extraction sheets. JM and RM conceived the research question. All the coauthors critically reviewed the protocol. NAA, LL and RM critically reviewed the search strategy and inclusion criteria. Finally, LL, AVR and RM critically reviewed the data extraction sheets. DMP is a guarantor for this work.

Funding The work on the protocol is the part of the programme of research 'Developing primary care services for stroke survivors' funded by the National Institute for Health Research (NIHR)'s Programme Grants for Applied Research Programme (grant reference number PTC-RP-PG-0213-20001).

Disclaimer The funder had no involvement in developing the protocol but approved the final submission. The views expressed are those of the author(s) and not necessarily those of the NHS, the NIHR or the Department of Health.

Competing interests None declared.

Provenance and peer review Not commissioned; externally peer reviewed.

Open Access This is an Open Access article distributed in accordance with the Creative Commons Attribution Non Commercial (CC BY-NC 4.0) license, which permits others to distribute, remix, adapt, build upon this work noncommercially, and license their derivative works on different terms, provided the original work is properly cited and the use is non-commercial. See: http:// creativecommons.org/licenses/by-nc/4.0/

\section{REFERENCES}

1. Gulliford MC, Charlton J, Rudd A, et al. Declining 1-year case-fatality of stroke and increasing coverage of vascular risk management: population-based cohort study. J Neurol Neurosurg Psychiatr 2010;81:416-22.
2. Lee S, Shafe ACE, Cowie MR. UK stroke incidence, mortality and cardiovascular risk management 1999-2008: time-trend analysis from the General Practice Research Database. BMJ Open 2011;1: e000269.

3. McKevitt C, Fudge N, Redfern J, et al. Self-reported long-term needs after stroke. Stroke 2011;42:1398-403.

4. National Audit Office. Progress in improving stroke care: Department of Health, HC 291, Report by Comptroller and Auditor General, Session 2009-10. Stationery Office, 2010. http://www.nao.org.uk/ report/department-of-health-progress-in-improving-stroke-care/\# (accessed 2 Feb 2016).

5. Murray J, Young J, Forster A. Review of longer-term problems after a disabling stroke. Rev Clin Gerontol 2007;17:277-92.

6. Allison R, Shelling $L$, Dennett $R$, et al. The effectiveness of various models of primary care-based follow-up after stroke: a systematic review. Prim Health Care Res Dev 2011;12:214-22.

7. Aziz NA, Leonardi-Bee J, Phillips M, et al. Therapy-based rehabilitation services for patients living at home more than one year after stroke. Cochrane Database Syst Rev 2008;(2): CD005952.

8. Care Quality Commission. Supporting life after stroke: a review of services for people who have had a stroke and their carers, 2011. http://www.cqc.org.uk/sites/default/files/documents/supporting_life after_stroke_national_report.pdf (accessed Jan 2011).

9. Royal College of Physicians, Sentinel Stroke National Audit Programme. How good is stroke care? First SSNAP annual report. Care received from April 2013 to March 2014. Royal College of Physicians, 2014. http://www.strokeaudit.org/Documents/Newspress/ SSNAP-Annual-Report-(April-2013-March-2014).pdf (accessed Oct 2015).

10. Adamson J, Beswick A, Ebrahim S. Is stroke the most common cause of disability? J Stroke Cerebrovasc Dis 2004;13:171-7.

11. Redfern J, McKevitt C, Wolfe CDA. Development of complex interventions in stroke care: a systematic review. Stroke 2006;37:2410-19.

12. Langhorne P, Bernhardt J, Kwakkel G. Stroke rehabilitation. Lancet 2011;377:1693-702.

13. Aziz N. Long-term rehabilitation after stroke: where do we go from here? Rev Clin Gerontol 2010;20:239-45.

14. Kwakkel G, Kollen B, Lindeman E. Understanding the pattern of functional recovery after stroke: facts and theories. Restor Neurol Neurosci 2004:22:281-99.

15. Lager KE, Mistri AK, Khunti K, et al. Interventions for improving modifiable risk factor control in the secondary prevention of stroke. Cochrane Database Syst Rev 2014;(5):CD009103.

16. MacKay-Lyons M, Thornton M, Ruggles T, et al. Non-pharmacological interventions for preventing secondary vascular events after stroke or transient ischemic attack. Cochrane Database Syst Rev 2013;(3):CD008656.

17. Forster A, Brown L, Smith J, et al. Information provision for stroke patients and their caregivers. Cochrane Database Syst Rev 2012; (11):CD001919.

18. Pollock A, Baer G, Campbell P, et al. Physical rehabilitation approaches for the recovery of function and mobility following stroke. Cochrane Database Syst Rev 2014;(4):CD001920.

19. Pollock A, Farmer SE, Brady MC, et al. Interventions for improving upper limb function after stroke. Cochrane Database Syst Rev 2014; (11):CD010820.

20. Campbell Burton CA, Holmes J, Murray J, et al. Interventions for treating anxiety after stroke. Cochrane Database Syst Rev 2011;(12) CD008860.

21. Hackett ML, Anderson CS, House A, et al. Interventions for preventing depression after stroke. Cochrane Database Syst Rev 2008;(3):CD003689.

22. Hackett ML, Yang M, Anderson CS, et al. Pharmaceutica interventions for emotionalism after stroke. Cochrane Database Syst Rev 2010;(2):CD003690.

23. Cheng HY, Chair SY, Chau JP. The effectiveness of psychosocial interventions for stroke family caregivers and stroke survivors: a systematic review and meta-analysis. Patient Educ Couns 2014;95:30-44.

24. Saban KL, Sherwood PR, DeVon HA, et al. Measures of psychological stress and physical health in family caregivers of stroke survivors: a literature review. $J$ Neurosci Nurs 2010;42:128-38.

25. Visser-Meily JM, Post MW, Riphagen II, et al. Measures used to assess burden among caregivers of stroke patients: a review. Clin Rehabil 2004;18:601-23.

26. Bowen A, Hazelton C, Pollock A, et al. Cognitive rehabilitation for spatial neglect following stroke. Cochrane Database Syst Rev 2013; (7):CD003586. Published Online First: 1 July 2013. 
27. Chung CS, Pollock A, Campbell T, et al. Cognitive rehabilitation for executive dysfunction in adults with stroke or other adult non-progressive acquired brain damage. Cochrane Database Syst Rev 2013;(4):CD008391. Published Online First: 30 April 2013.

28. Royal College of General Practitioners (RCGP). A 2022 GP: a vision for general practice in the future NHS. London: RCGP, 2013.

29. Rashidian A, Shakibazadeh E, Karimi-Shahanjarini A, et al. Barriers and facilitators to the implementation of doctor-nurse substitution strategies in primary care: qualitative evidence synthesis. Cochrane Database Syst Rev 2013:CD010412. doi:10.1002/14651858. CD010412.

30. Wiley-Exley E. Evaluations of community mental health care in lowand middle-income countries: a 10-year review of the literature. Soc Sci Med 2007;64:1231-41.

31. Foot C, Sonola L, Bennett L, et al. Managing quality in community health care services. London: The King's Fund, 2014. http://www. kingsfund.org.uk/sites/files/kf/field/field_publication_file/managingquality-in-community-health-care-services.pdf (accessed Dec 2014).

32. Andrews GR, Faulkner D, Andrews M, et al. Ageing and Health Technical Report: A glossary of terms for community health care and services for older persons. 2004. http://www.who.int/kobe_centre/ ageing/ahp_vol5_glossary.pdf. (accessed Oct 2015).

33. Fearon P, Langhorne P. Services for reducing duration of hospital care for acute stroke patients. Cochrane Database Syst Rev 2012; (9):CD000443.

34. Barnett K, Mercer SW, Norbury M, et al. Epidemiology of multimorbidity and implications for health care, research, and medical education: a cross-sectional study. Lancet 2012; 380:37-43.

35. Stroke Association. State of the Nation: stroke statistics. London, UK: Stroke Association, 2016. https://www.stroke.org.uk/resources/ state-nation-stroke-statistics (accessed 29 June 2015).

36. Royal College of Physicians, Clinical Effectiveness and Evaluation Unit on behalf of the Intercollegiate Stroke Working Party. Sentinel Stroke National Audit Programme (SSNAP): Clinical audit AprilJune 2015 public report: Sentinel Stroke National Audit Programme (SSNAP), 2015. https://www.strokeaudit.org/Documents/Results/ National/AprJun2015/AprJun2015-PublicReport.aspx (accessed June 2015)

37. Brereton L, Carroll C, Barnston S. Interventions for adult family carers of people who have had a stroke: a systematic review. Clin Rehabil 2007;21:867-84.

38. Gaugler JE. The longitudinal ramifications of stroke caregiving: a systematic review. Rehabil Psychol 2010;55:108-25.

39. McGurk R, Kneebone II. The problems faced by informal carers to people with aphasia after stroke: a literature review. Aphasiology 2013;27:765-83.

40. Salter K, Zettler L, Foley N, et al. Impact of caring for individuals with stroke on perceived physical health of informal caregivers. Disabil Rehabil 2010;32:273-81.

41. White CL, Lauzon S, Yaffe MJ, et al. Toward a model of quality of life for family caregivers of stroke survivors. Qual Life Res 2004;13:625-38.

42. Laver KE, George S, Thomas S, et al. Virtual reality for stroke rehabilitation. Cochrane Database Syst Rev 2015;(2): CD008349.

43. Stroke Association. Feeling overwhelmed: the emotional impact of stroke. Stroke Association, 2013.

44. Stroke Association. State of the Nation: Stroke Statistics, 2015. http://www.stroke.org.uk/sites/default/files/stroke_statistics_2015.pdf (accessed 29 Jun 2015).

45. Higgins J, Green S, eds. Cochrane handbook for systematic reviews of interventions. The Cochrane Collaboration, 2011. http://www. cochrane-handbook.org (accessed Oct 2015).
46. Farace DJ, Frantzen J. Sixth international conference on grey literature: work on grey in progress. Grey literature 2004 conference proceedings. Amsterdam: TextRelease, 2004.

47. Arksey H, O'Malley L. Scoping studies: towards a methodological framework. Int J Soc Res Methodol 2005;8:19-32.

48. The Joanna Briggs Institute. Joanna Briggs Institute Reviewers' Manual: 2015 edition/Supplement. Adelaide: The Joanna Briggs Institute, 2015. http://joannabriggs.org/assets/docs/sumari/ Reviewers-Manual_Methodology-for-JBI-Scoping-Reviews_2015_v2. pdf (accessed Oct 2015)

49. Aziz NA, Pindus DM, Mullis R, et al. Understanding stroke survivors' and informal carers' experiences of and need for primary care and community health services-a systematic review of the qualitative literature: protocol. BMJ Open 2016;6:e009244.

50. International Labour Office. International Standard Classification of Occupations ISCO-08. Volume 1: Structure, group definitions and correspondence tables. Geneva: International Labour Office, 2012. http://www.ilo.org/public/english/bureau/stat/isco/ (accessed $20 \mathrm{Dec}$ 2015).

51. Geyh S, Cieza A, Schouten J, et al. ICF Core Sets for stroke. $J$ Rehabil Med 2004:135-41.

52. Levac D, Colquhoun H, O'Brien K. Scoping studies: advancing the methodology. Implement Sci 2010;5:69.

53. Centre for Reviews and Dissemination (CRD). Systematic Reviews: CRD's guidance for undertaking reviews in healthcare. York: York Publishing Services Ltd, 2009. http://www.york.ac.uk/crd/SysRev/! SSL!/WebHelp/1_3_UNDERTAKING_THE_REVIEW.htm (accessed Oct 2015).

54. Balshem $\mathrm{H}$, Stevens $\mathrm{A}$, Ansari M. Finding grey literature evidence and assessing for outcome and analysis reporting biases when comparing medical interventions: $A H R Q$ and the effective health care program. Rockville, MD: Agency for Healthcare Research and Quality (US), 2013. http://www.ncbi.nlm.nih.gov/books/NBK174882/

55. Mahood Q, Van Eerd D, Irvin E. Searching for grey literature for systematic reviews: challenges and benefits. Res Synth Methods 2014;5:221-34.

56. Duffield A, Reid G, Walker D, et al. Review of the published literature for the impact and costeffectiveness of six nutrition related emergency interventions ENN, 2004. http://www.ennonline.net/ reviewsixnutritioninterventions (accessed 15 Oct 2015)

57. Godin K, Stapleton J, Kirkpatrick S, et al. Applying systematic review search methods to the grey literature: a case study examining guidelines for school-based breakfast programs in Canada. Syst Rev 2015;4:138

58. Thomson S, Osborn R, Squires D, et al. International profiles of health care systems, 2012 Australia, Canada, Denmark, England, France, Germany, Iceland, Italy, Japan, the Netherlands, New Zealand, Norway, Sweden, Switzerland, and the United States. New York, NY: The Commonwealth Fund, 2012. http://apps.who.int/ medicinedocs/documents/s19988en/s19988en.pdf (accessed Oct 2015).

59. Scottish Intercollegiate Guidelines Network (SIGN). Search filters Healthcare Improvement Scotland, 2014. http://www.sign.ac.uk methodology/filters.html (accessed 20 Aug 2015).

60. Robinson KA, Dickersin K. Development of a highly sensitive search strategy for the retrieval of reports of controlled trials using PubMed. Int J Epidemiol 2002;31:150-3.

61. Lefebvre C, Manheimer E, Glanville J. Chapter 6: Searching for studies. In: Higgins JP, Green S, eds. Cochrane Handbook for Systematic Reviews of Interventions. Version 5.1.0 (updated March 2011). The Cochrane Collaboration. http://handbook.cochrane.org/ chapter_6/box_6_4_a_cochrane_hsss_2008_sensmax_pubmed.htm

62. McKibbon KA, Wilczynski NL, Haynes RB. Retrieving randomized controlled trials from Medline: a comparison of 38 published search filters. Health Info Libr J 2009;26:187-202. 